\title{
Force Prediction in Ultrasonic Vibration-Assisted Milling
}

\section{Yixuan Feng ${ }^{1,}$, Fu-Chuan $\mathrm{Hsu}^{2}$, Yu-Ting Lu ${ }^{2}$, Yu-Fu Lin ${ }^{2}$, Chorng-Tyan Lin $^{2}$, Chiu-Feng Lin²,} Ying-Cheng $\mathrm{Lu}^{2}$, Steven Y. Liang ${ }^{1}$

1. Woodruff School of Mechanical Engineering, Georgia Institute of Technology, Atlanta, GA, 30332

2. Metal Industries Research and Development Centre (MIRDC), Kaohsiung, Taiwan

*Corresponding Author: Yixuan Feng yfeng82@gatech.edu

Co-authors:

Fu-Chuan Hsu fchsu@ mail.mirdc.org.tw

Yu-Ting Lu lyting@mail.mirdc.org.tw Yu-Fu Lin yufulin@mail.mirdc.org.tw

Chorng-Tyan Lin chontyan@mail.mirdc.org.tw Chiu-Feng Lin chiufeng@ mail.mirdc.org.tw

Ying-ChengLuycl@mail.mirdc.org.tw_Steven Y. Liang steven.liang@me.gatech.edu

Abstract: Force reduction is one of the most important benefit of applying ultrasonic vibration on milling. However, most of studies so far are limited to experimental investigation. In the current study, an analytical predictive model on cutting forces in ultrasonic vibration-assisted milling is proposed. The three types of toolworkpiece criteria are considered based on the instantaneous position and velocity of tool center. Type I criterion indicates that there is no contact if the instantaneous velocity is opposite to tool rotation direction. Type II criterion checks whether the vibration displacement is larger than the instantaneous uncut chip thickness. Type III criterion considers the overlaps between current and previous tool paths due to vibration. If none of these criteria is satisfied, milling forces are nonzero. Then the calculation is performed by transforming milling and tool geometry configuration to orthogonal cutting at each instant. The orthogonal cutting forces are predicted through the exhaustive search of shear angle and calculation of shear flow stress on tool-chip interface. The axial force is then calculated based on tool geometry, and the milling forces in feed, cutting, and axial directions are calculated after coordinate transformation. The proposed predictive force model in ultrasonic vibration-assisted milling is validated through comparison to experimental measurements on Aluminum alloy 2A12. The predicted values are able to match the measured milling forces with high accuracy of average difference of $13.6 \%$ in feed direction and $13.8 \%$ in cutting direction.

Keywords: force; ultrasonic vibration; modeling; milling

\section{Introduction}

Non-conventional milling has been developed for years in order to reach high precision, extend tool life while keep the material removal rate. Ultrasonic vibration-assisted milling is a newly developed non-conventional milling process to achieve these targets in a more ecofriendly manner comparing to laser-assisted or electrical discharge milling. Micro-scale high frequency vibration with small amplitude is applied on the tool or workpiece to realize the tool-workpiece separation during the milling process. Several research studies have concluded that the tool-workpiece separation in ultrasonic vibration-assisted milling is the main reason for several benefits including improved surface quality [1], lower machining forces, and extended tool life [2]. This tool-workpiece 
interaction is microscopically non-monotonic which facilitates chip separation and therefore reduces machining forces. In addition, the frequent separation reduces the deformation zone of workpiece during milling which is the main reason for improved surface finish. Several experimental investigations and statistical analysis have been conducted on ultrasonic vibration-assisted milling. Hsu et al. [3] analyzed the effects of milling parameters on cutting forces in ultrasonic vibration-assisted milling of Inconel 718 and found that depth of cut was the dominant factor. Ibrahim et al. [4] studied the cutting force reduction on Aluminium A16061, and a 32\% reduction was recorded on mean forces while peak forces were at same level for conventional and ultrasonic vibrationassisted milling. Similarly, Halim et al. [5] recorded a 10\% reduction on maximum machining force in feed direction for ultrasonic vibration-assisted milling of carbon fiber reinforced plastic. These studies reflect the benefits of ultrasonic vibration-assisted milling, but the quantitative conclusions are limited to specific material and process parameter combinations, and are unable to reveal the physics nature analytically.

Up to now, there are few analytical models proposed for force prediction in ultrasonic vibration-assisted milling. Verma et al. [6] predicted static machining force in axial ultrasonic vibration-assisted milling. The instantaneous chip thickness is calculated based on the frequency and maximum velocity of vibration, and the mean cutting force for the specific tool angular position is derived from contact ratio and shear flow stress based on JohnsonCook model. This method is able to calculate mean oblique cutting forces accurately through mechanics of machining. However, the tool-workpiece separation criterion in axial direction is relatively easy to decide, and the dynamics of moving tool or workpiece is simplified as a contact ratio. In addition, Ding et al. [7] predicted cutting forces in two-dimensional vibration-assisted micro milling. Shen et al. [8] calculated cutting force of micro end milling with ultrasonic vibration in normal direction. Zarchi et al. [9] predicted cutting forces by analytically determining the critical cutting speed and tool-workpiece separation zones. Elhami et al. [10] also proposed analytical model on cutting force in two-dimensional vibration-assisted milling including a heat transfer model to incorporate the thermal effect. Later, Verma et al. [11] predicted machining forces with ultrasonic vibration through analysis of variance and regression equations. All the models so far are either not fully analytical or limited in specific vibration direction. In current study, an analytical force predictive model is proposed which is able to characterize vibration in three directions and decide three types of tool-workpiece separation criteria. The previously proposed model [12-18] predicts the milling forces based on instantaneous tool rotation angle, feed rate, cutting speed, and axial depth of milling. At each specific tool angular position, the milling is considered as equivalent orthogonal cutting, and all cutting as well as tool geometry parameters are recalculated. The strain and strain rate are decided based on contact mechanics [19], and the flow stress is calculated dependent on strain, strain rate, and temperature [20]. The machining forces are predicted by stress over contact area, and the coordinate transformation is applied to disassemble forces into feed, cutting, and axial directions. The force predictive model in ultrasonic vibration-assisted milling is validated through comparison of experimental measurements on Aluminum alloy 2A12 [21, 22]. A sensitivity analysis is also conducted to estimate average forces under the effects of different cutting and ultrasonic parameters including axial depth of milling, feed per tooth, ultrasonic frequency, and spindle rotation frequency. 


\section{Force predictive model in ultrasonic vibration-assisted milling}

\subsection{Tool-workpiece separation criteria}

Kinematical analysis is conducted to calculate the exact trajectory of tool center as well as tip under ultrasonic vibration, tool rotation, and feed movement. According to the coordinate system in Fig. 1, when the initial tool center position is chosen as origin, the tool center is described as a function of time as

$x(t)=A_{x} \sin (\omega t)-f t$

$y(t)=A_{y} \sin (\omega t)$

$z(t)=A_{z} \sin (\omega t)-d$

where $f$ is the feed rate, $d$ is axial depth of milling, $\omega=2 \pi f_{0}, f_{0}$ is the ultrasonic vibration frequency, $A_{x}$, $A_{y}$, and $A_{z}$ are the vibration amplitude in each direction, which is zero when no vibration is applied on that direction. The tool center velocity is then calculated based on the derivative of equations (1)-(3) as

$$
\begin{aligned}
& x^{\prime}(t)=\omega A_{x} \cos (\omega t)-f \\
& y^{\prime}(t)=\omega A_{y} \cos (\omega t) \\
& z^{\prime}(t)=\omega A_{z} \cos (\omega t)
\end{aligned}
$$

In addition,

$$
\begin{aligned}
& V_{x}(t)=\omega A_{x} \cos (\omega t)-f-V_{r} \cos \left(\phi_{r}\right) \\
& V_{y}(t)=\omega A_{y} \cos (\omega t)+V_{r} \sin \left(\phi_{r}\right)
\end{aligned}
$$

describe the velocity of tool tip. $V_{r}$ is the cutting speed dependent on spindle rotation frequency and cutter size, and $\phi_{r}$ is rotation angle.

There are three types of tool-workpiece separation criteria that decide whether there is contact between tool and workpiece at the instant. The first two types are described by equations (1)-(8). Type I criterion is satisfied when the cutting direction component of the relative velocity between tool and workpiece is opposite to the tool rotation direction $[1,23]$. As shown in Fig. 2(a), $V_{n}=\sqrt{V_{x}^{2}+V_{y}^{2}}$ is the resultant cutting velocity based on equations (7) and (8), while $V_{u l}=z^{\prime}(t)$ is the ultrasonic vibration velocity in axial direction, $\beta$ is the helix angle. In Fig. 2(b), the component along the transverse direction decides the type I intermittent effect at it is perpendicular to the uncut surface. When this component is negative, which is oppose to the tool rotation direction, there is no contact between tool and workpiece as described by

Type I criterion: $V_{n} \cos (\beta)-V_{u l} \sin (\beta)<0$

Type II criterion decides whether vibration displacement in the instantaneous cutting thickness direction (i.e., 
tool radial direction) is larger than the instantaneous uncut chip thickness. If the tool center is at a position where it is further away from the workpiece comparing to the initial position, tool and workpiece are separated even though Type I criterion is not satisfied. The criterion is mathematically expressed as

Type II criterion: $x(t)>0$ or $y(t)>0$

Type III criterion happens when the current tool path with vibration assistance overlaps in some regions with the surface contour left by previous cutting path(s). As shown in Fig. 3(a), in conventional milling, the chip is continuous and has large thickness. While in ultrasonic vibration-assisted milling, much smaller and shorter chips are produced because of the overlaps between cutting paths as shown in Fig. 3(b). Therefore, the average radial chip thickness is recalculated considering the extra displacement from previous cutting path due to vibration as

Type III criterion: $\bar{t}_{U V A, \text { radial }}=\bar{t}_{c}-\frac{1}{2} \frac{\omega A_{x} \cos (\omega t)}{R P M / 60}$

where RPM is the spindle rotation frequency. It is assumed that there is no phase difference between two cutting paths.

Therefore, before the prediction of machining forces, the tool center trajectory is predicted, and the toolworkpiece separation criteria are checked. If there is no contact, the machining forces are zero. If there is contact, the forces are calculated based on instantaneous axial depth of milling $z(t)$, feed rate $x^{\prime}(t)$, and cutting speed $V_{r}$

\subsection{Instantaneous equivalent cutting and geometry parameters with ultrasonic vibration}

At each specific tool angular position, the milling is considered as equivalent orthogonal cutting, and all cutting as well as tool geometry parameters are recalculated. The average radial depth of cut or chip thickness is given as

$\overline{t_{c}}=\frac{1}{2} \frac{x^{\prime}(t)}{R P M / 60}$

The tangential chip thickness is calculated as

$$
\bar{t}_{U V A, \text { tangential }}=\pi D \times \frac{R P M / 60}{f_{0}}
$$

where $D$ is the tool diameter. Then the instantaneous equivalent cutting depth is calculated based on equations (11) and (13) by average chip thickness in two directions as

$$
t_{U V A}=\sqrt{2} \times \frac{\bar{t}_{U V A, \text { radial }}+\bar{t}_{U V A, \text { tangential }}}{2} \times \sin \left(\phi_{r}\right) \times \cos C_{s}^{*}
$$

where the equivalent side cutting edge angle $C_{s}{ }^{*}$ is defined as 
$C_{s}^{*}=C_{s}+\eta_{c}$

The chip flow angle $\eta_{c}$ is calculated based on tool geometry and cutting parameters [13].

Then the equivalent chip flow angle $\eta_{c}{ }^{*}$ and the equivalent inclination angle $i^{*}$ are

$\eta_{c}^{*}=i^{*}=\arcsin \left(\cos \eta_{0} \sin i-\sin \eta_{0} \sin \alpha \cos i\right)$

where $i$ is the inclination angle, $\alpha$ is the rake angle, $\eta_{0}$ is defined as

$$
\eta_{0}=\arccos \left(\frac{\sec i-\tan i \tan \eta_{c} \tan \alpha}{\sqrt{\left(\tan i-\tan \eta_{c} \tan \alpha \sec i\right)^{2}+\sec ^{2} \eta_{c}}}\right)
$$

The equivalent rake angle is

$$
\alpha^{*}=\arcsin \left(\frac{\sec \eta_{0} \sin i-\sin i^{*}}{\tan \eta_{0} \cos i^{*}}\right)
$$

The cutting width in orthogonal cutting is related to axial depth of milling as,

$$
w^{*}=\frac{z(t)}{\cos \left(C_{s}^{*}\right)}
$$

\subsection{Orthogonal cutting forces with ultrasonic vibration}

As shown in Fig. 4, the shear length along shear plane $\mathrm{AB}$ is given by

$$
l_{s}=\frac{t_{U V A}}{\sin \phi}
$$

where shear angle $\phi$ is decided through exhaustive search. For chip speed or the shear velocity $V_{s}$, it is derived from cutting speed as

$$
V_{s}=\frac{\cos \alpha^{*}}{\cos \left(\phi-\alpha^{*}\right)} \sqrt{V_{x}^{2}+V_{y}^{2}}
$$

The plastic strain rate and strain are then calculated

$\dot{\varepsilon}_{A B}=\frac{C_{O x l e y} V_{s}}{\sqrt{3} l_{s}}$

$\varepsilon_{A B}=\frac{\cos \alpha^{*}}{2 \sqrt{3} \sin \phi \cos \left(\phi-\alpha^{*}\right)}$

where $C_{O x l e y}$ is a model coefficient. The average temperature in the shear plane is described as

$T_{A B}=T_{0}+\eta \Delta T$ 
where $T_{0}$ is the room temperature, $\eta$ is the plastic energy to enthalpy conversion ratio. The temperature increment is due to the shear energy in the primary shear plane $F_{s} V_{s}$, the chip flow rate is $\rho \sqrt{V_{x}^{2}+V_{y}^{2}} t_{U V A} w^{*}$. The temperature rise in the primary shear zone is obtained as [12]

$$
\Delta T=\frac{(1-\beta) F_{s} V_{s}}{C_{P} \rho \sqrt{V_{x}^{2}+V_{y}^{2}} t_{U V A} w^{*}}
$$

where $F_{s}$ is the shear force, $F_{s}=k_{A B} \times l_{s} \times w^{*}, \beta$ is the energy dissipation coefficient, $\rho$ is the material density, $C_{P}$ is the heat capacity. The average material flow stress in the shear plane is given by Johnson-Cook model as $k_{A B}=\frac{1}{\sqrt{3}}\left(A+B \varepsilon_{A B}{ }^{n}\right)\left(1+C \ln \frac{\dot{\varepsilon}_{A B}}{\dot{\varepsilon}_{0}}\right)\left(1-\left(\frac{T_{A B}-T_{w}}{T_{m}-T_{w}}\right)^{m}\right)$

where A, B, C, m, n are model parameters based on material properties, $\dot{\varepsilon}_{0}$ is the reference strain rate, $T_{m}$ is the melting temperature of workpiece. The values of the parameters (A, B, C, m, and n) for the Aluminum alloy are $243.0,618.8,0.01,1.6$, and 0.2 , respectively [24]. The angle $\theta$ between resultant force $\mathrm{R}$ and shear plane $\mathrm{AB}$ is defined as:

$\theta=\tan ^{-1}\left(1+2\left(\frac{\pi}{4}-\phi\right)-C_{n}\right)$

where $C_{n}=C_{\text {Oxley }} n \frac{B \varepsilon_{A B}^{n}}{A+B \varepsilon_{A B}^{n}}$. The friction angle $\lambda$ is denoted as

$$
\lambda=\theta+\alpha^{*}-\phi
$$

The tool and chip contact length is

$$
h=\frac{t_{U V A} \sin \theta}{\cos \lambda \sin \phi}\left(1+\frac{C n}{3 \tan \theta}\right)
$$

By assuming the uniform stress distribution along the chip tool interface, the shear stress is calculated as

$$
\tau_{\text {int }}=\frac{F}{h w^{*}}
$$

where $F$ is the shear force, $F=\frac{F_{S}}{\cos \theta} \sin \lambda$. With a similar approach, the temperature at the tool and chip interface is

$$
T_{\mathrm{int}}=\frac{F \sin \alpha^{*}}{\rho C_{p} t_{U V A} w^{*} \cos \left(\phi-\alpha^{*}\right)}+T_{A B}
$$

The strain and strain rate in the chip are 


$$
\begin{aligned}
& \varepsilon_{\mathrm{int}}=2 \varepsilon_{A B}+\frac{h}{\sqrt{3} \delta t_{d}} \\
& \dot{\varepsilon}_{\mathrm{int}}=\frac{V_{C}}{\sqrt{3} \delta t_{d}}
\end{aligned}
$$

where $t_{d}$ is the deformed chip thickness and $\delta$ is a coefficient related to the thickness of plastic deformation zone on tool chip interface. Then the average flow stress in chip is

$$
k_{\text {chip }}=\frac{1}{\sqrt{3}}\left(A+B \varepsilon_{i n t}^{n}\right)\left(1+C \ln \frac{\dot{\varepsilon}_{\text {int }}}{\dot{\varepsilon}_{0}}\right)\left(1-\left(\frac{T_{i n t}-T_{w}}{T_{m}-T_{w}}\right)^{m}\right)
$$

Through the exhaustive search of $\phi, C_{O x l e y}$ and $\delta$, these parameters are decided when $\tau_{\text {int }}=k_{\text {chip }}$ and the largest shear angle $\phi$ is selected. The orthogonal cutting forces are then calculated as

$$
\begin{aligned}
& F_{c}=\frac{F_{s}}{\cos \theta} \cos \left(\lambda-\alpha^{*}\right) \\
& F_{t}=\frac{F_{s}}{\cos \theta} \sin \left(\lambda-\alpha^{*}\right)
\end{aligned}
$$

\subsection{Milling forces with ultrasonic vibration}

After the cutting force $F_{c}$ and radial force $F_{t}$ being calculated, the axial force is calculated as

$$
F_{r}=\frac{F_{c}\left(\sin i^{*}-\cos i^{*} \sin \alpha^{*} \tan \eta_{c}^{*}\right)-F_{t} \cos \alpha^{*} \tan \eta_{c}{ }^{*}}{\sin i^{*} \sin \alpha^{*} \tan \eta_{c}{ }^{*}+\cos i^{*}}
$$

These three forces are first transferred into cutting, feed, and axial directions as

$$
\begin{aligned}
& P_{1}=F_{c} \\
& P_{2}=-F_{t} \cos \left(C_{S}^{*}\right)-F_{r} \sin \left(C_{S}^{*}\right) \\
& P_{3}=F_{t} \sin \left(C_{S}^{*}\right)-F_{r} \cos \left(C_{S}^{*}\right)
\end{aligned}
$$

Then, the forces are transferred into Cartesian coordinates as

$$
\begin{aligned}
& F_{x}(\phi)=P_{1} \cos \left(\phi_{r}\right)+P_{2} \sin \left(\phi_{r}\right) \\
& F_{y}(\phi)=-P_{1} \sin \left(\phi_{r}\right)-P_{2} \cos \left(\phi_{r}\right) \\
& F_{z}(\phi)=-P_{3}
\end{aligned}
$$

\section{Validation by experimental data}

The proposed predictive force model in ultrasonic vibration-assisted milling is validated through comparison to experimental measurements on Aluminum alloy 2A12 [21, 22]. The experiments are performed on five-axis 
high-speed CNC machining center. The ultrasonic generator and vibrator convert high-frequency oscillation electric energy into workpiece vibration along the feed direction. The dynamic cutting force signals are gathered through dynamometer. The cutting tool is a two-flute end mill made of cemented carbide. The diameter is $2 \mathrm{~mm}$, and the helix angle is $30^{\circ}$. The feed per tooth is $3 \mu \mathrm{m}$, the spindle rotation frequency is $1,000 \mathrm{r} / \mathrm{min}$, and the axial depth of milling is $0.2 \mathrm{~mm}$. For ultrasonic vibration, the vibration frequency $f_{0}$ is $19.58 \mathrm{kHz}$, and four slotmilling experiments are conducted with vibration amplitude of 0 (conventional milling), 4, 6, and $8 \mu \mathrm{m}$, respectively, as shown in Fig. 5.

The machining forces in feed and cutting directions are calculated in analytical model between a rotation angle of $0^{\circ}$ and $180^{\circ}$. If the tool-workpiece separation criterion is met, both forces are recorded as zero. Otherwise, the forces are calculated based on methodology described in section two. The original predicted force profiles are shown in Fig. 6 with ten data points within one ultrasonic vibration period. The number of force data points predicted is later reduced to accommodate the number of measurements limited by the sampling frequency of dynamometer. The values of the parameters (A, B, C, m, and n) for the Aluminum alloy are 243.0, 618.8, 0.01, 1.6, and 0.2, respectively [24]. Fig. 7 shows single-sided amplitude spectrums of measured as well as predicted $F_{x}$ and $F_{y}$ when vibration amplitude is $8 \mu \mathrm{m}$ in half cutting cycle. The sampling frequency is $1,750 \mathrm{~Hz}$ for dynamometer. The fast Fourier transform of both experimental and predicted data shows presence of vibration around $1,750 \mathrm{~Hz}$ due to intermittent cutting effect under the sampling frequency. Another peak is observed at 0 $\mathrm{Hz}$ occurring due to tool engagement. The predicted spectrums in Fig. 7 (b) and (d) have more low frequency components since forces are considered zero when there is no contact between tool and workpiece, while measured force signals still have nonzero values at valleys. In addition, measured spectrums in Fig. 7 (a) and (c) have more high frequency components as a result of noise such as tool chatter. Overall, the predicted force amplitude spectrums have good agreements with experimental measurements on both feed and cutting forces.

Fig. 8 shows the average cutting forces from experiments and predictive model under four different vibration amplitudes. The average forces are 1.93 and $1.7 \mathrm{~N}$ for conventional milling in feed and cutting directions. With a vibration amplitude of $4 \mu \mathrm{m}$, the average forces in two directions are 1.17 and $1.1 \mathrm{~N}$, respectively. The average values decrease by $39 \%$ in feed direction and $35 \%$ in cutting direction when the ultrasonic vibration is applied. In addition, the measured forces keep dropping gradually when the amplitude increases, since the tool-workpiece separation time is longer under higher vibration amplitude. The drop rate is approximately a constant as the average forces decrease linearly. The feed force is changing from 1.17 to 1.09 followed by $1.05 \mathrm{~N}$ as ultrasonic vibration amplitude increases from 4 to 6 and $8 \mu \mathrm{m}$. Similarly, the cutting force is changing from 1.1 to 1.05 followed by $0.98 \mathrm{~N}$ as ultrasonic vibration amplitude increases from 4 to 6 and $8 \mu \mathrm{m}$.

The predicted average forces are plotted as dashed lines in Fig. 8. For conventional milling, both predicted forces are higher than measurements. In feed direction, the predicted value is $2.01 \mathrm{~N}$ which is $4.15 \%$ higher. In cutting direction, the prediction is $2.14 \mathrm{~N}$ and the error is $25.88 \%$ which is acceptable comparing with previous established conventional milling force predictive model [13]. When the ultrasonic vibration is applied, the 
average values decrease by $50 \%$ in feed direction and $35 \%$ in cutting direction. The feed force is changing from 1.01 to 0.96 followed by $0.79 \mathrm{~N}$ as ultrasonic vibration amplitude increases from 4 to 6 and $8 \mu \mathrm{m}$. Similarly, the cutting force is changing from 1.39 to 1.07 followed by $0.97 \mathrm{~N}$ as ultrasonic vibration amplitude increases from 4 to 6 and $8 \mu \mathrm{m}$. The maximum error in all cases is less than 27\%. Overall, the proposed force prediction model is able to match the trend with average error of $13.6 \%$ in $F_{x}$ and $13.8 \%$ in $F_{y}$.

\section{Sensitivity analysis}

In order to appreciate the proposed predictive model, sensitivity analysis is conducted to estimate average forces under the effects of different cutting and ultrasonic parameters including axial depth of milling, feed per tooth, ultrasonic frequency, and spindle rotation frequency. The ultrasonic vibration amplitude is fixed at $6 \mu \mathrm{m}$, and other parameters are the same as in section three. As shown in Fig. 9 (a), a higher axial depth of milling will significantly increase the milling forces in both directions. $F_{x}$ and $F_{y}$ are doubled from 0.96 and $1.07 \mathrm{~N}$ to 1.92 and $2.14 \mathrm{~N}$ when the axial depth of milling changes from 0.2 to $0.4 \mathrm{~mm}$. The axial depth of milling decides the cutting width according to equation (19). With the increase of cutting width, the contact area is also expanded resulting in higher forces under same flow stress. Similarly, a doubled feed per tooth from 3 to $6 \mu \mathrm{m}$ also approximately doubles $F_{x}$ and $F_{y}$ from 0.96 and $1.07 \mathrm{~N}$ to 1.88 and $2.20 \mathrm{~N}$ because of higher radial cutting depth according to equation (12), as shown in Fig. 9 (b). On the other hand, tangential chip thickness is smaller when the ultrasonic vibration frequency increases as in equation (13), and the cutting speed is higher, leading to decreased milling forces. $F_{x}$ and $F_{y}$ are $25.4 \%$ and $15.0 \%$ smaller when the ultrasonic vibration frequency increases by $28.6 \%$ as depicted in Fig. 9 (c). The spindle rotation frequency also decides cutting speed through spindle speed. $F_{x}$ and $F_{y}$ are $28.7 \%$ and $13.2 \%$ smaller when the spindle rotation frequency increases by $250 \%$ as depicted in Fig. 9 (d). The results of sensitivity analysis are in good agreement with conclusions from Verma et al. [6].

\section{Conclusion}

In current study, an analytical predictive model on cutting forces in ultrasonic vibration-assisted milling is proposed. The three types of tool-workpiece criteria are considered based on the instantaneous position and velocity of tool center. Type I criterion indicates that there is no contact if the instantaneous velocity is opposite to tool rotation direction. Type II criterion checks whether the vibration displacement is larger than the instantaneous uncut chip thickness. Type III criterion considers the overlaps between current and previous tool paths due to vibration. If none of these criteria is satisfied, milling forces are nonzero. Then the calculation is performed by transforming milling and tool geometry configuration to orthogonal cutting at each instant. The orthogonal cutting forces are predicted through the exhaustive search of shear angle and calculation of shear flow stress on tool-chip interface. The axial force is then calculated based on tool geometry, and the milling forces in feed, cutting, and axial directions are calculated after coordinate 
transformation. The proposed predictive force model in ultrasonic vibration-assisted milling is validated through comparison to experimental measurements on Aluminum alloy 2A12. The following conclusions are summarized

- The average forces in ultrasonic vibration-assisted milling are significantly lowered by over $35 \%$ comparing to conventional milling.

- The average forces keep decreasing as the vibration amplitude increases, since the tool-workpiece separation time is longer and the effective cutting time is shorter.

- The proposed predictive model is able to match the measured milling forces with high accuracy of average difference of $13.6 \%$ in feed direction and $13.8 \%$ in cutting direction.

- Based on the sensitivity analysis, a doubled axial depth of milling or feed per tooth will double the milling forces in both directions, while a higher ultrasonic vibration or spindle rotation frequency will result in lower milling forces.

The proposed method is up to now the first approach to predict forces in milling with ultrasonic vibration in feed direction. The model can be expended and applied in milling with vibration in any direction. It is valuable in terms of providing an accurate and reliable reference for the prediction of milling forces in ultrasonic vibration-assisted milling.

\section{References}

1. Chen, W., et al., State-of-the-art review on vibration-assisted milling: principle, system design, and application. The International Journal of Advanced Manufacturing Technology, 2018. 97(5-8): p. 2033-2049.

2. $\mathrm{Xu}, \mathrm{W} . \mathrm{X}$. and L.-C. Zhang, Ultrasonic vibration-assisted machining: principle, design and application. Advances in Manufacturing, 2015. 3(3): p. 173-192.

3. Hsu, C.Y., et al., A Study on Ultrasonic Vibration Milling of Inconel 718. Key Engineering Materials, 2009. 419420: p. 373-377.

4. Rasidi, I., et al., A performance of 2 dimensional ultrasonic vivration assisted milling in cutting froce reduction, on Aluminium AL6061 ARPN Journal of Engineering and Applied Sciences, 2016. 11(18).

5. Halim, N.F.H.A., H. Ascroft, and S. Barnes, Analysis of Tool Wear, Cutting Force, Surface Roughness and Machining Temperature During Finishing Operation of Ultrasonic Assisted Milling (UAM) of Carbon Fibre Reinforced Plastic (CFRP). Procedia Engineering, 2017. 184: p. 185-191.

6. Verma, G.C., P.M. Pandey, and U.S. Dixit, Modeling of static machining force in axial ultrasonic-vibration assisted milling considering acoustic softening. International Journal of Mechanical Sciences, 2018. 136: p. 1-16.

7. Ding, H., S.J. Chen, and K. Cheng, Two-dimensional vibration-assisted micro end milling: Cutting force modelling and machining process dynamics. Proceedings of the Institution of Mechanical Engineers, Part B: Journal of Engineering Manufacture, 2010. 224(12): p. 1775-1783.

8. Shen, X.H., et al., A Study on Cutting Force in Micro End Milling with Ultrasonic Vibration. Advanced Materials Research, 2010. 97-101: p. 1910-1914.

9. Abootorabi Zarchi, M.M., M.R. Razfar, and A. Abdullah, Investigation of the effect of cutting speed and vibration amplitude on cutting forces in ultrasonic-assisted milling. Proceedings of the Institution of Mechanical Engineers, Part B: Journal of Engineering Manufacture, 2012. 226(7): p. 1185-1191.

10. Elhami, S., M.R. Razfar, and M. Farahnakian, Analytical, numerical and experimental study of cutting force 
during thermally enhanced ultrasonic assisted milling of hardened AISI 4140. International Journal of Mechanical Sciences, 2015. 103: p. 158-171.

11. Verma, G.C. and P.M. Pandey, Machining forces in ultrasonic-vibration assisted end milling. Ultrasonics, 2019. 94: p. 350-363.

12. Pan, Z., et al., Turning Force Prediction of AISI 4130 Considering Dynamic Recrystallization. 2017(50725): p. V001T02A040.

13. Pan, Z., et al., Force modeling of Inconel 718 laser-assisted end milling under recrystallization effects. The International Journal of Advanced Manufacturing Technology, 2017. 92(5): p. 2965-2974.

14. Pan, Z., et al., Microstructure-sensitive flow stress modeling for force prediction in laser assisted milling of Inconel 718. Manufacturing Rev., 2017. 4: p. 6.

15. Feng, Y., et al., Inverse analysis of the cutting force in laser-assisted milling on Inconel 718. The International Journal of Advanced Manufacturing Technology, 2018. 96(1): p. 905-914.

16. Ning, J., V. Nguyen, and S.Y. Liang, Analytical modeling of machining forces of ultra-fine-grained titanium. The International Journal of Advanced Manufacturing Technology, 2018.

17. Mirkoohi, E., P. Bocchini, and S.Y. Liang, An analytical modeling for process parameter planning in the machining of Ti-6Al-4V for force specifications using an inverse analysis. The International Journal of Advanced Manufacturing Technology, 2018. 98(9): p. 2347-2355.

18. Mirkoohi, E., P. Bocchini, and S.Y. Liang, Inverse analysis of residual stress in orthogonal cutting. Journal of Manufacturing Processes, 2019. 38: p. 462-471.

19. Ning, J. and S.Y. Liang, Inverse identification of Johnson-Cook material constants based on modified chip formation model and iterative gradient search using temperature and force measurements. The International Journal of Advanced Manufacturing Technology, 2019. 102(9): p. 2865-2876.

20. Mirkoohi, E., P. Bocchini, and S.Y. Liang, Analytical temperature predictive modeling and non-linear optimization in machining. The International Journal of Advanced Manufacturing Technology, 2019. 102(5): p. 1557-1566.

21. Shen, X.-H. and G.-F. Xu, Study of milling force variation in ultrasonic vibration-assisted end milling. Materials and Manufacturing Processes, 2017. 33(6): p. 644-650.

22. Shen, X.-H., et al., Ultrasonic vibration-assisted milling of aluminum alloy. The International Journal of Advanced Manufacturing Technology, 2012. 63(1-4): p. 41-49.

23. Abdur-Rasheed, A., A Fundamental Study of Vibration Assisted Machining. Advanced Materials Research, 2011. 264-265: p. 1702-1707.

24. Zhang, X., et al., Microstructure investigation and mechanical property analysis in electromagnetic riveting. The International Journal of Advanced Manufacturing Technology, 2014. 78(1-4): p. 613-623.

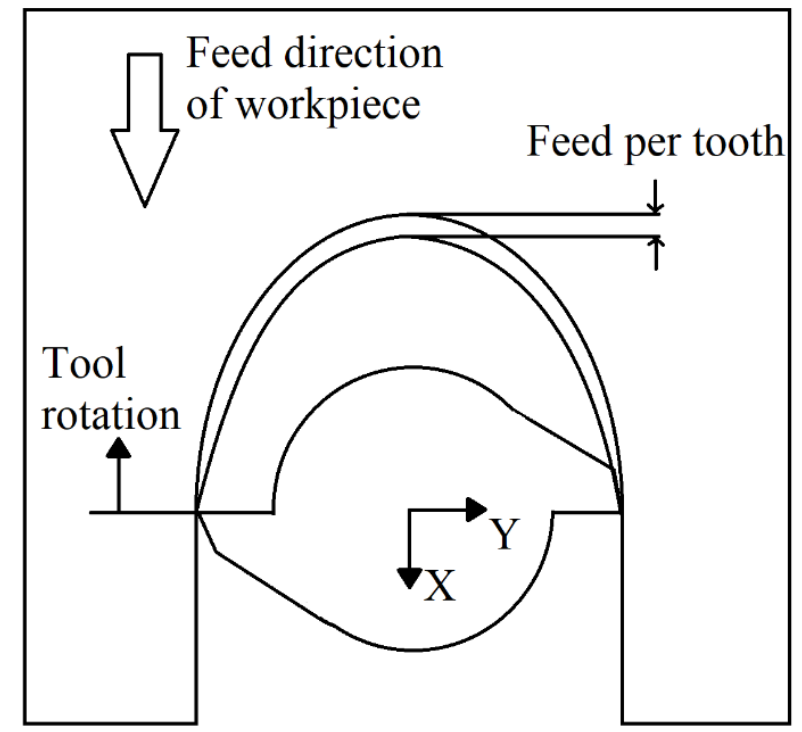

Fig.1 Coordinate system of cutting tool and workpiece 


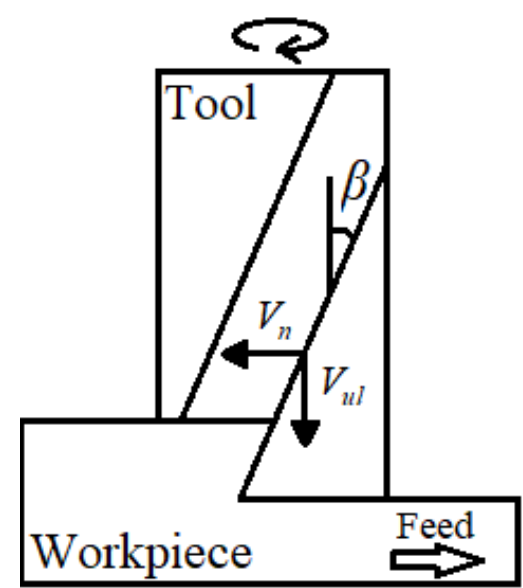

(a)

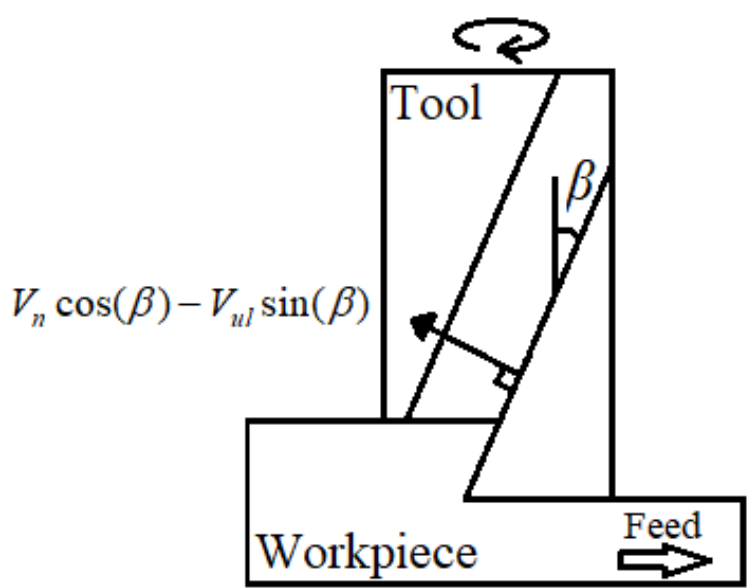

(b)

Fig.2 Velocity at the tool tip due to tool rotation and ultrasonic vibration: (a) representation of $V_{n}$ and $V_{u l}$ at the cutting tip (b) resolved component at the tip

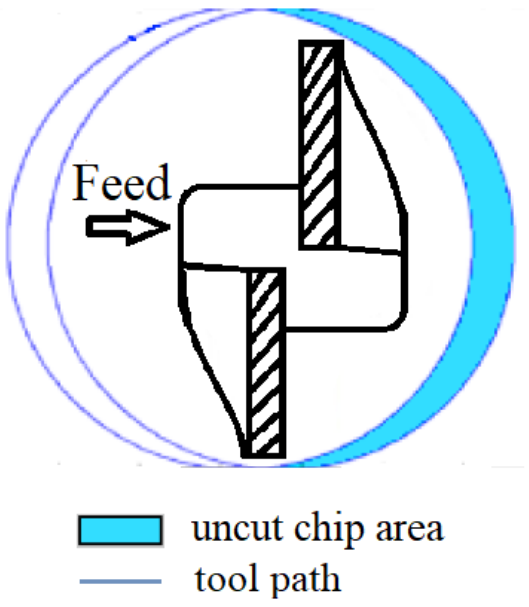

(a)

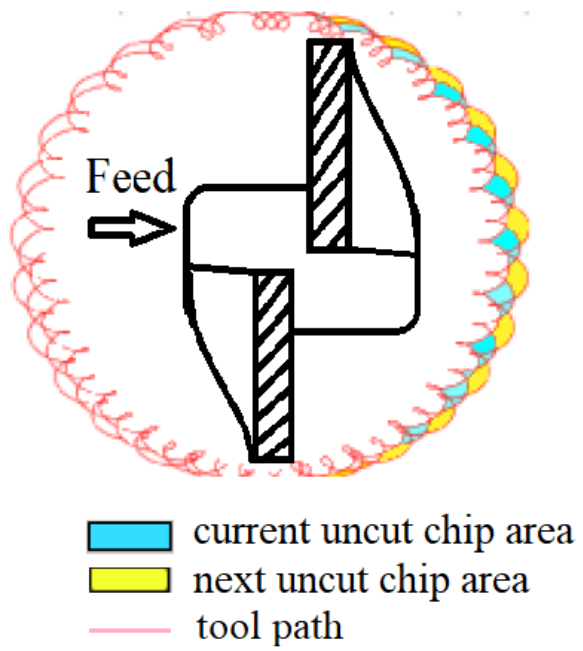

(b)

Fig.3 Difference of chip thickness affecting cutting force reduction in (a) conventional and (b) vibrationassisted milling 


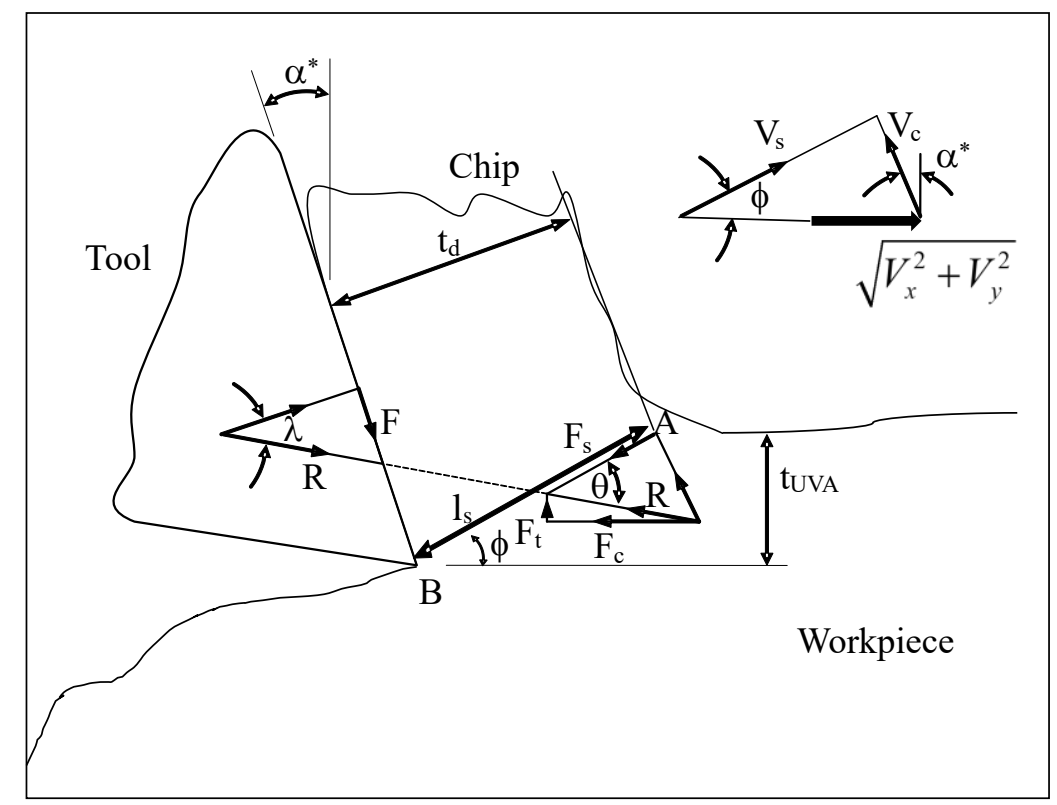

Fig. 4 Model of chip formation

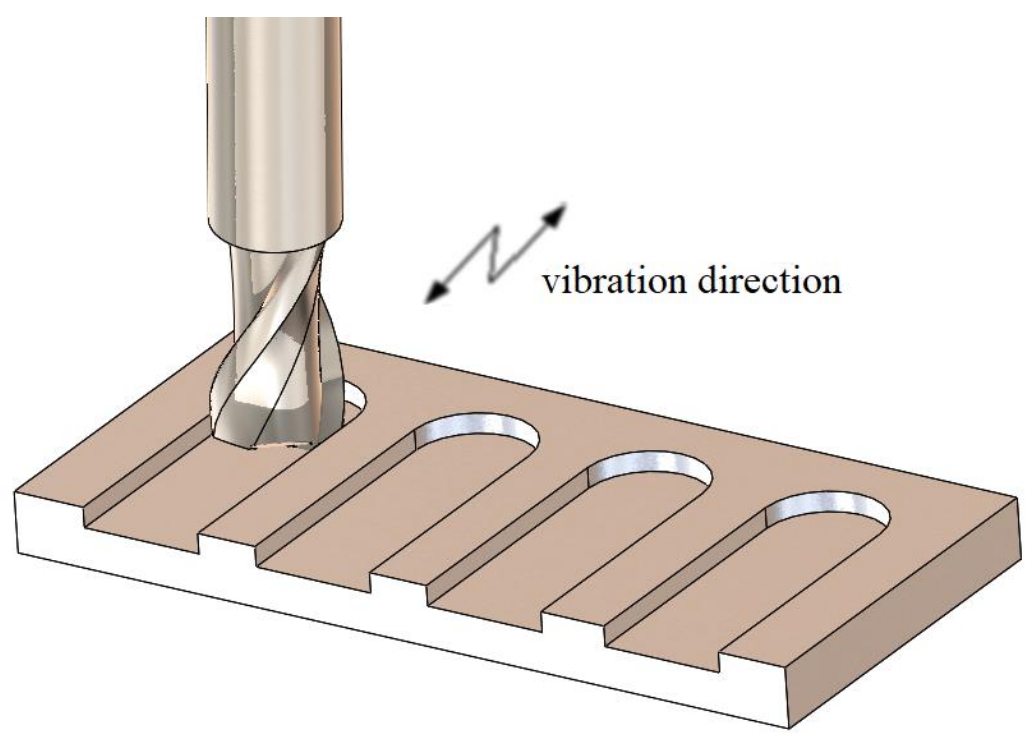

Fig. 5 Schematic of slot-milling process 


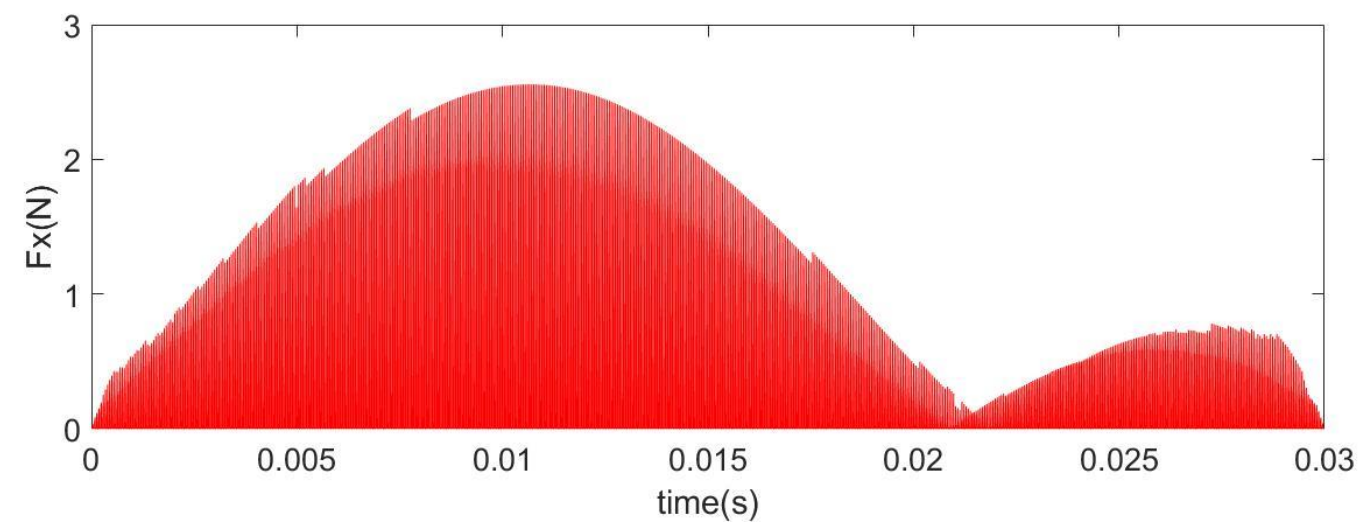

(a)

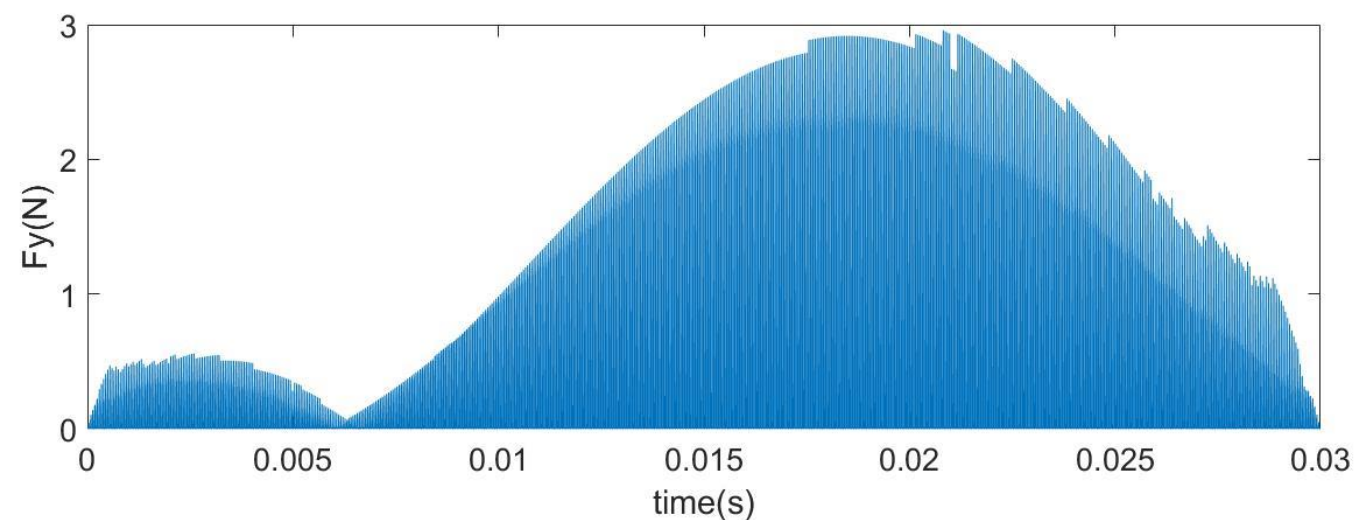

(b)

Fig. 6 Predicted force profiles of (a) $F_{x}$ and (b) $F_{y}$ when vibration amplitude is $8 \mu \mathrm{m}$ in half cutting cycle

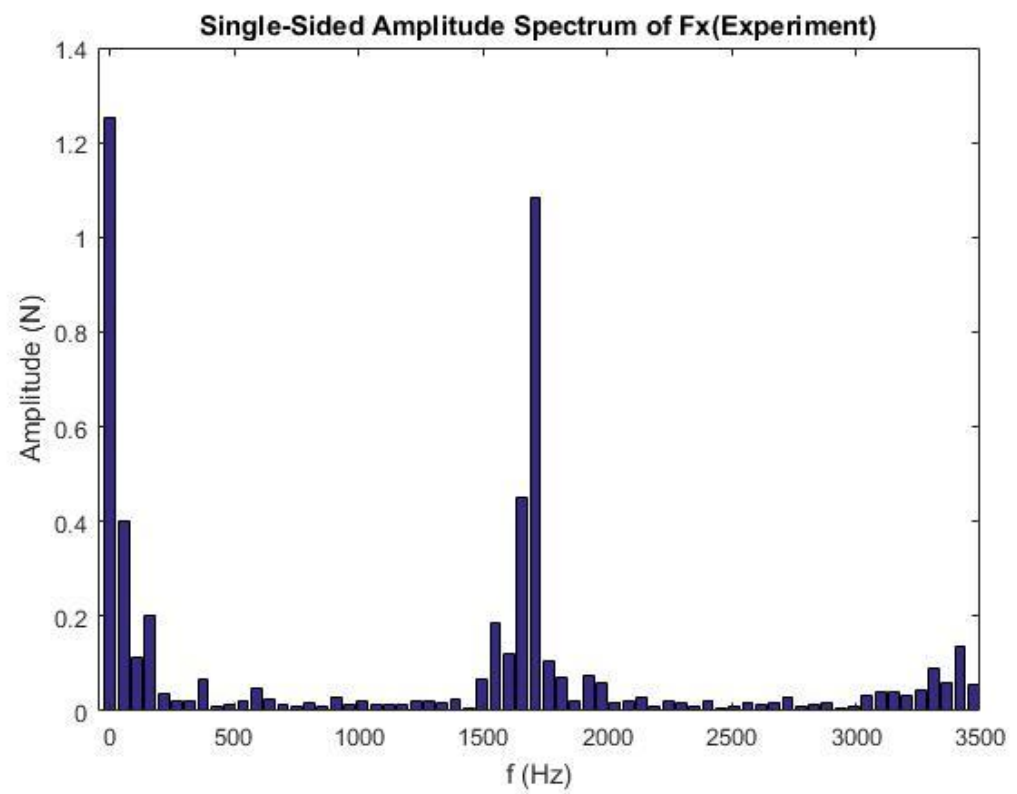

(a) 


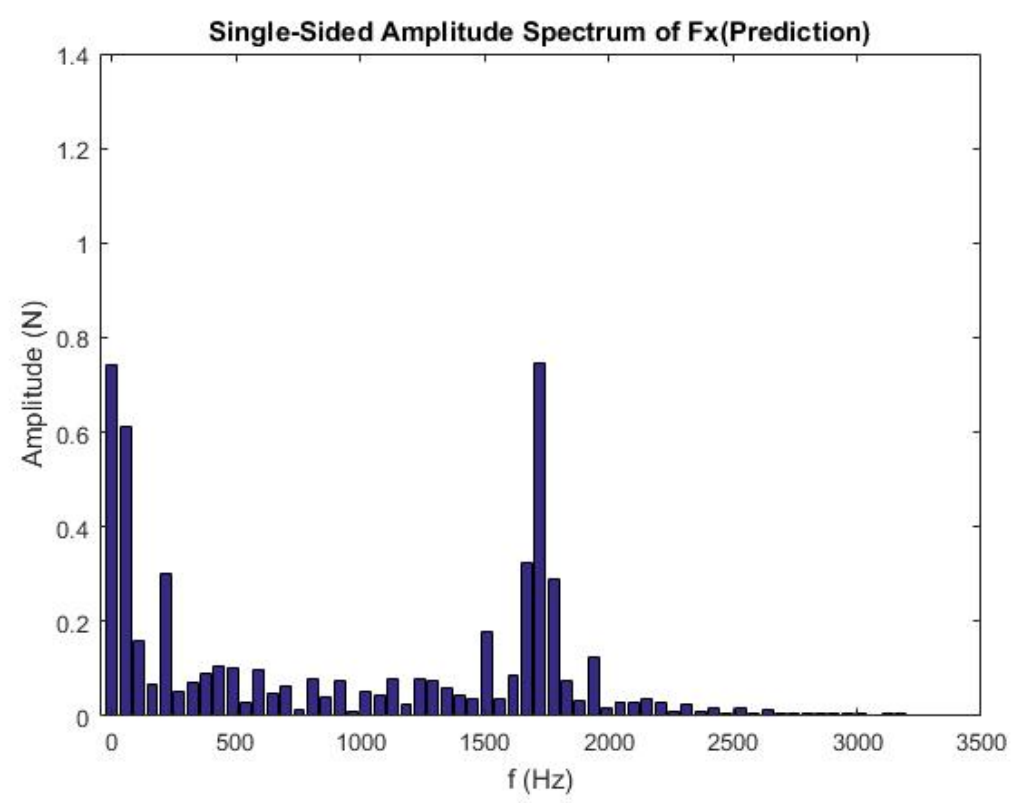

(b)

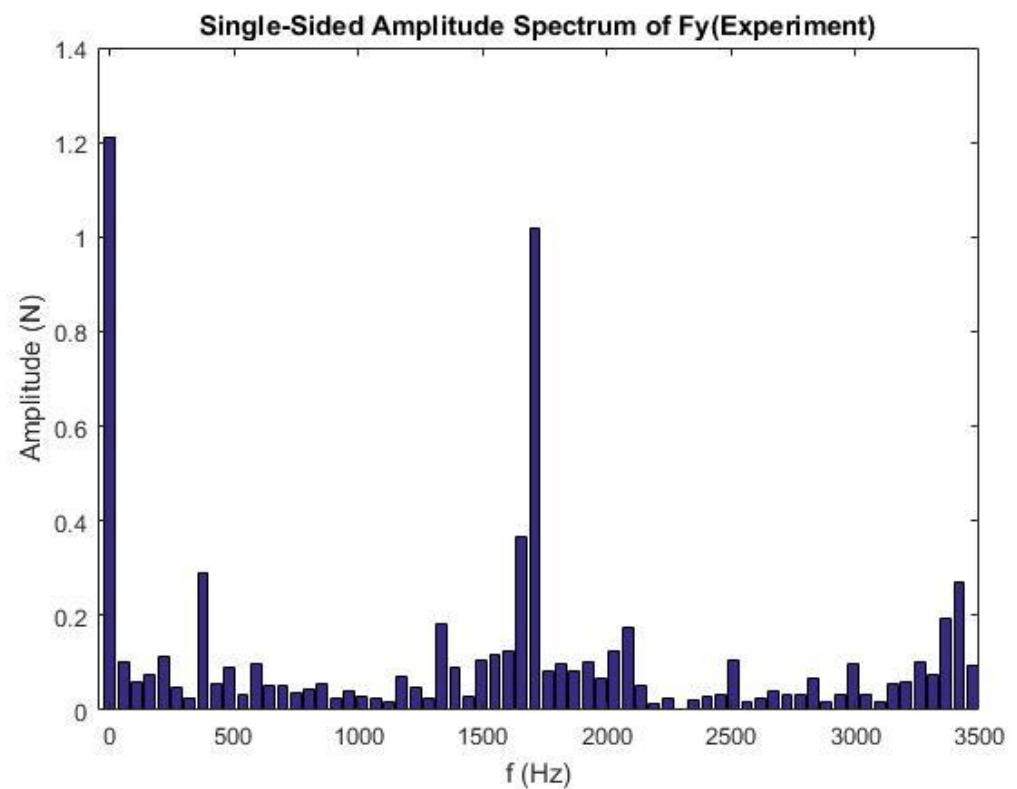

(c) 


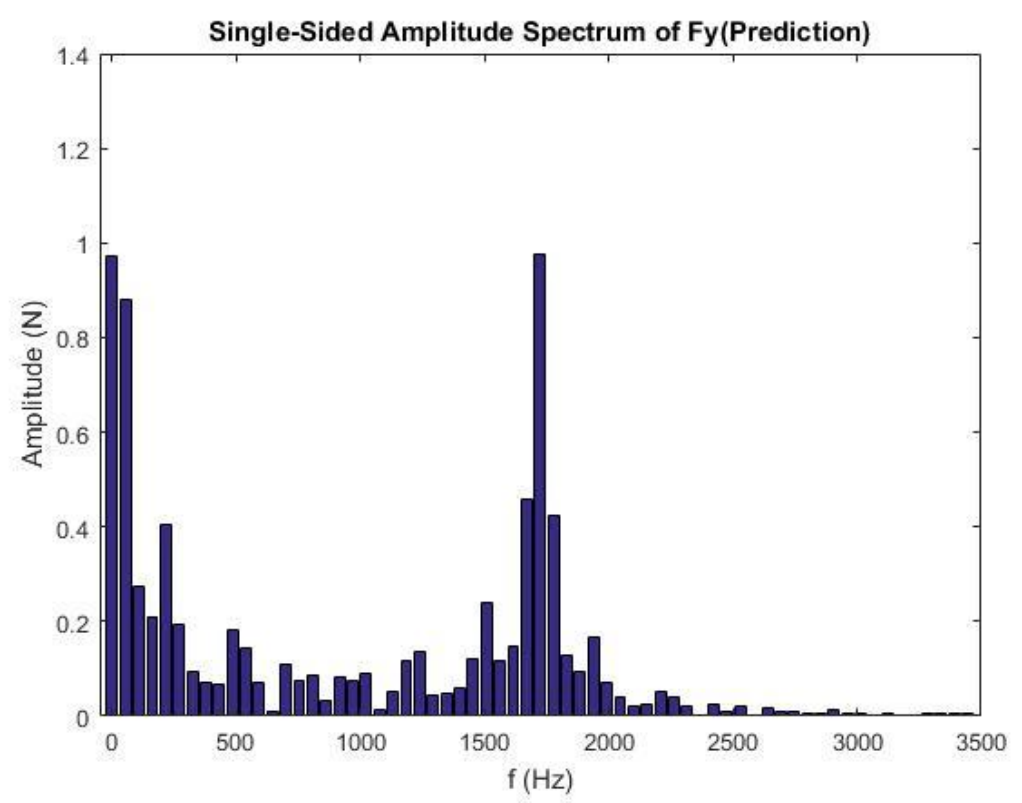

(d)

Fig. 7 Comparison of single-sided amplitude spectrum of $F_{x}$ between (a) experiment and (b) predictive model, $F_{y}$ between (c) experiment and (d) predictive model, during ultrasonic vibration-assisted milling with $8 \mu \mathrm{m}$ amplitude [22]

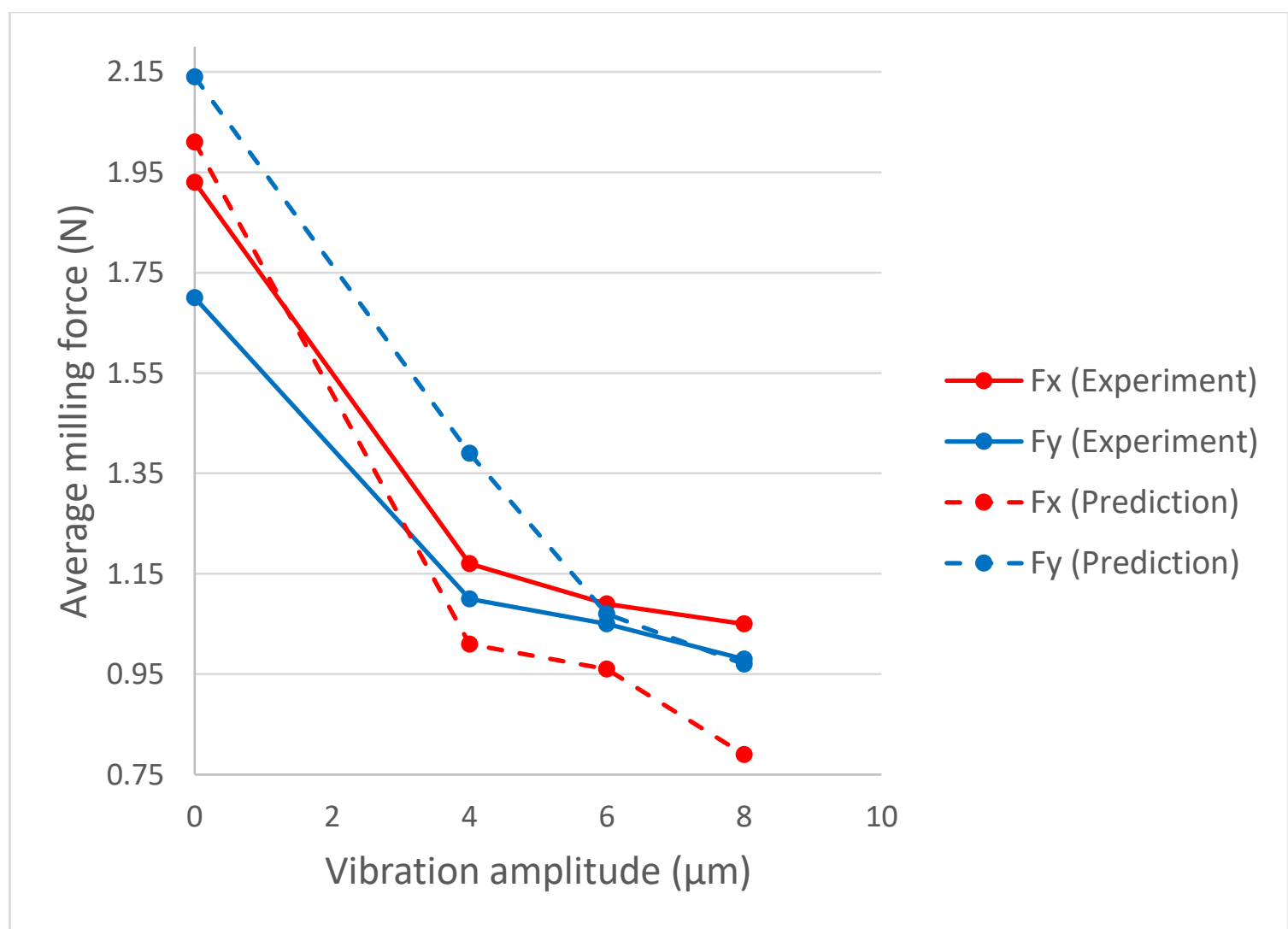

Fig. 8 Comparison of average milling forces with different vibration amplitudes [22] 


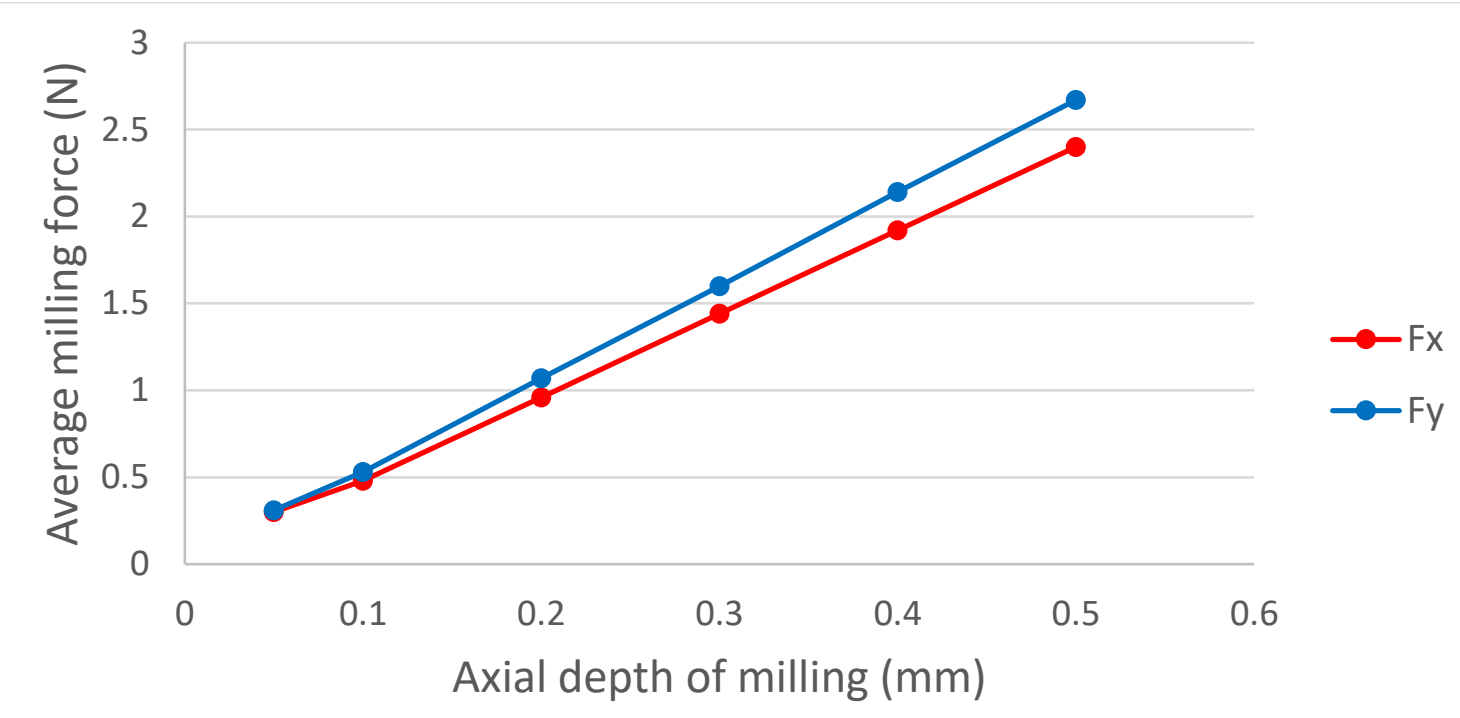

(a)

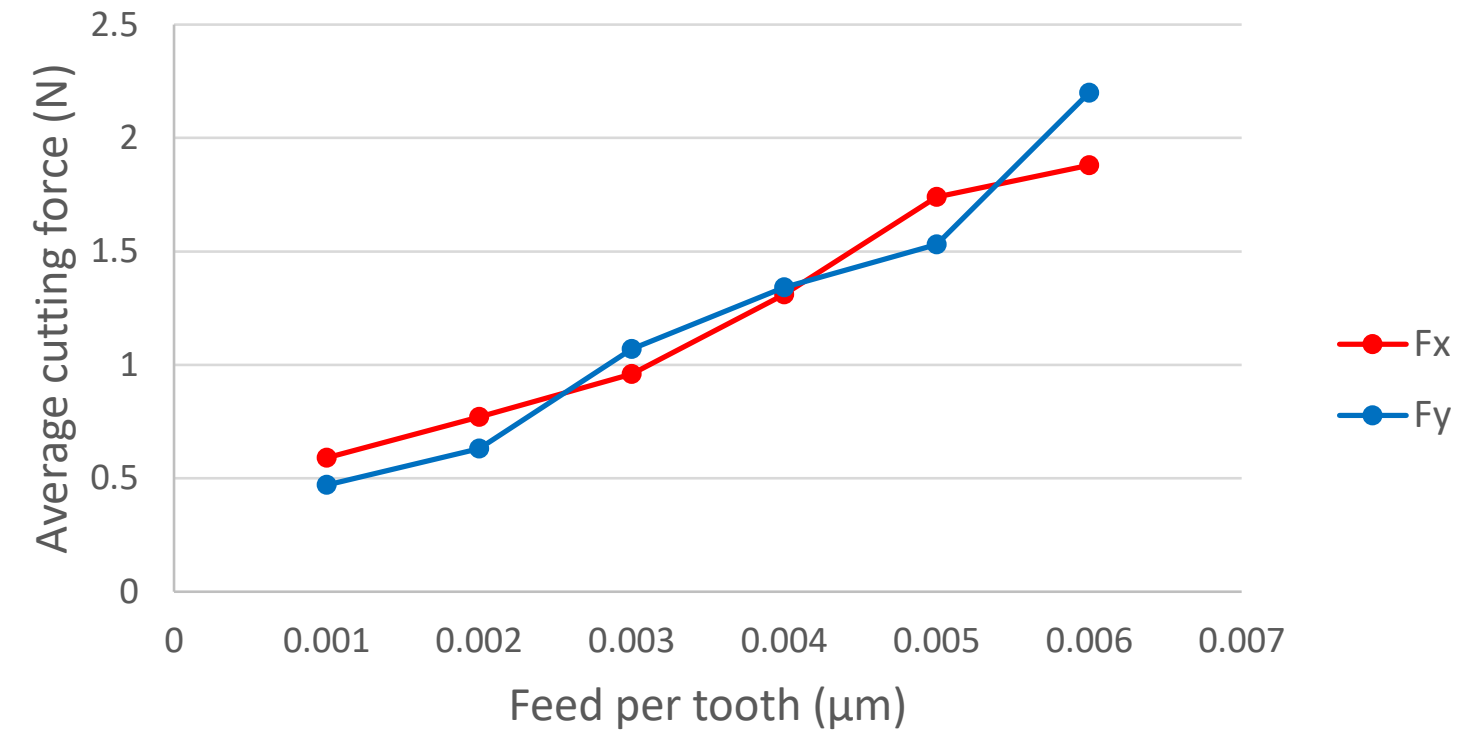

(b) 


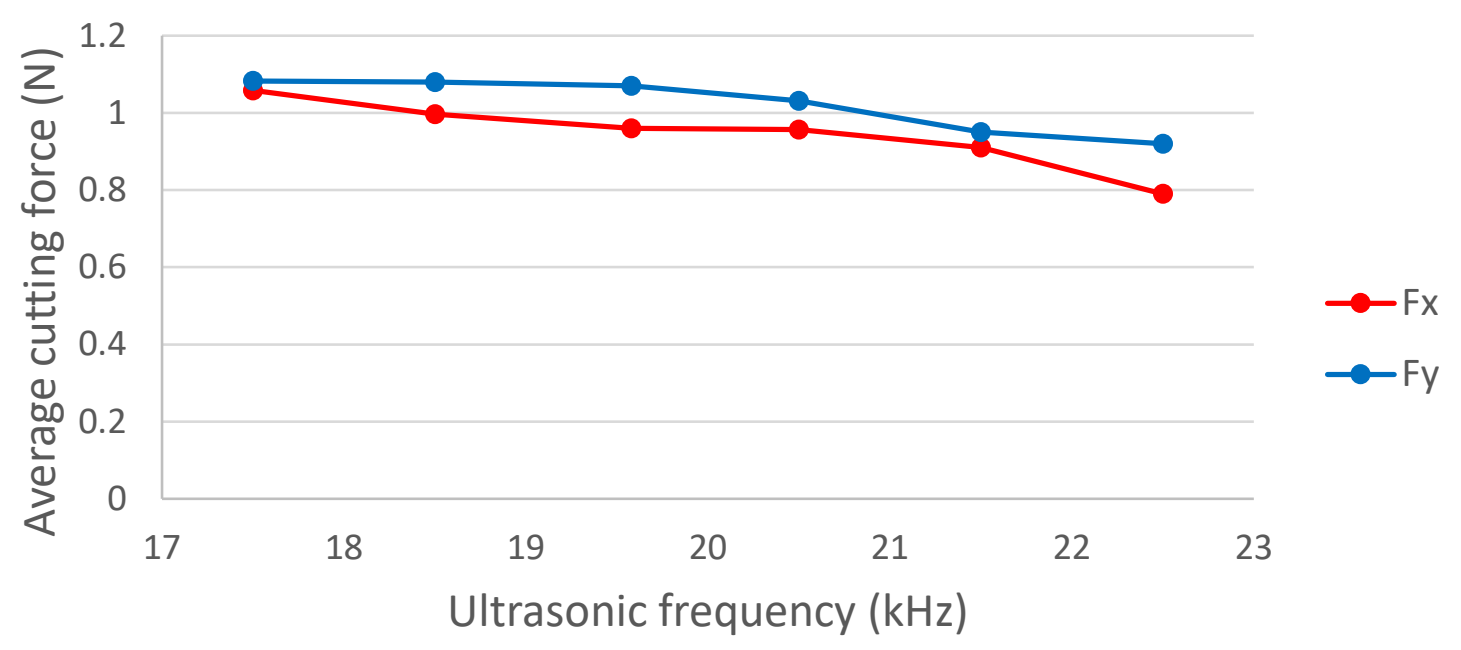

(c)

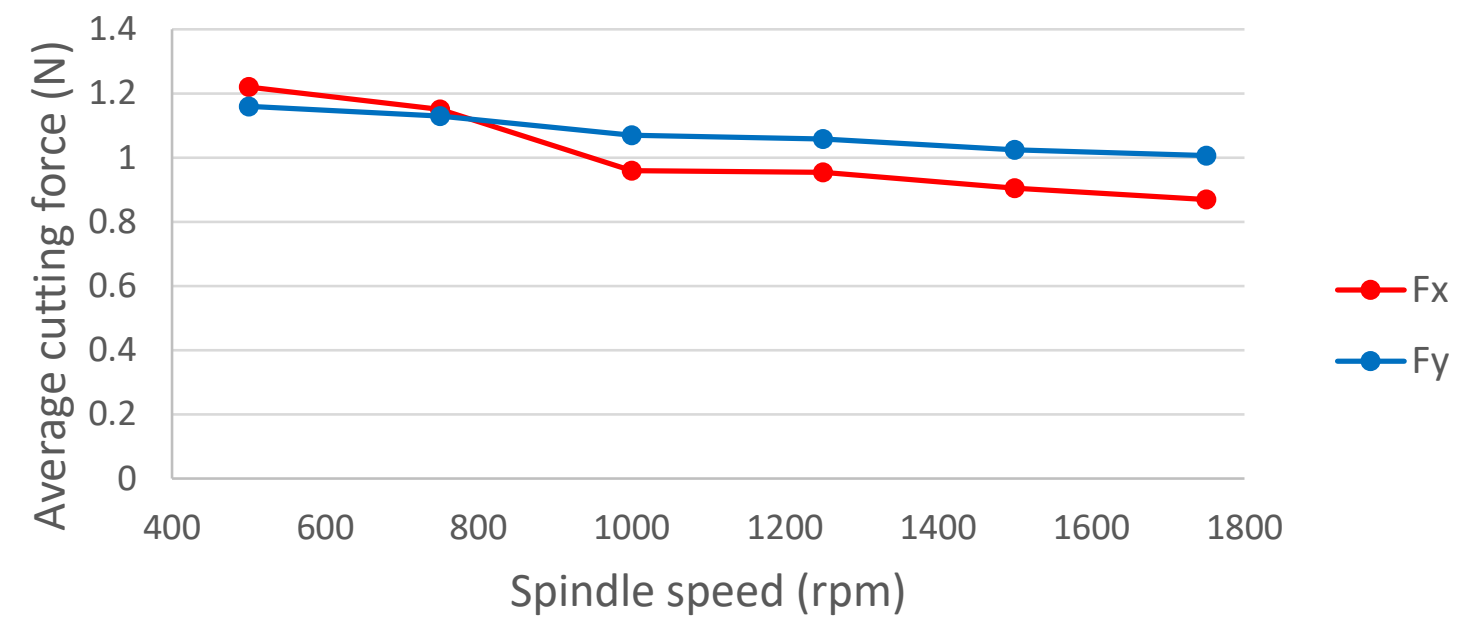

(d)

Fig. 9 The change of predicted forces under the effect of different cutting and vibration parameters (a) axial depth of milling (b) feed per tooth (c) ultrasonic frequency (d) spindle rotation frequency 